\title{
For a Science of Group Interaction
}

\author{
Gerry Stahl \\ Drexel University \\ College of Information Science \\ Philadelphia, PA, USA \\ $+1-215-895-0544$ \\ Gerry@GerryStahl.net
}

\begin{abstract}
As a foundation for the design of groupware, we need a new science of group interaction, a systematic description of the processes at the group level of description that may contribute to problem solving, knowledge building and other cognitive tasks undertaken by small groups collaborating synchronously over networked computers. A scientific investigation of the knowledge-building interactions of online teams involves explorations along multiple dimensions: (a) designing a testbed to support interaction within teams, (b) analyzing how interaction takes place within this setting and (c) describing how the teams achieve their tasks. This paper discusses how a current CSCL project designed a groupware environment in which this could take place and be studied; it reviews how the project approached the rigorous study of what took place there; and it reflects on the nature of group interaction as an object for a new science.
\end{abstract}

\section{Categories and Subject Descriptors}

H.1.1. Information Systems; Models and Principles; Systems and Information Theory; General Systems Theory. H.5.3. Group and Organizational Interfaces; Collaborative Computing and Computer-supported Cooperative Work.

\section{General Terms}

Theory.

\section{Keywords}

CSCL, CSCW, group cognition, group interaction.

\section{THE NEED FOR A NEW SCIENCE}

The design of software to support group work, knowledge building and problem solving should be built on the foundation of an understanding of the nature of group interaction. This paper argues that previous research in CSCW and CSCL is based on a collection of incommensurable theories, which are not grounded in an explicit investigation of group interaction. What is needed is a science of group interaction focused on the group level of description to complement psychological theories of individuals and social theories of communities.

Preparing for a new science requires three major undertakings:

- The domain of the science must not only be defined, it must be

Permission to make digital or hard copies of all or part of this work for personal or classroom use is granted without fee provided that copies are not made or distributed for profit or commercial advantage and that copies bear this notice and the full citation on the first page. To copy otherwise, or republish, to post on servers or to redistribute to lists, requires prior specific permission and/or a fee.

GROUP'09, May 10-13, 2009, Sanibel Island, Florida, USA.

Copyright 2009 ACM 978-1-60558-500-0/09/05...\$5.00. explored and captured in the form of a data corpus.

- Methods for analyzing the data must be selected, adapted, refined and mastered.

- Analytic findings must be organized in terms of a framework of theoretical conceptualizations.

After discussing the need for a new science of group interaction, this paper describes a research project that successfully approached these tasks by:

- Creating a synchronous online service in which small groups of students engaged in problem-solving work in mathematics,

- Conducting chat interaction analysis of a number of case studies from the data recorded in that service and

- Conceptualizing some of the features of the small-group interactions that were observed.

The focus on small groups was originally motivated by the realization that $\mathrm{CSCW}$ and $\mathrm{CSCL}$ are fundamentally different from other domains of study. They take as their subject matter cooperative working or collaborative learning, that is, what takes place when small groups of workers or students engage together in cognitive activities like problem solving or knowledge building [26, ch. 11]. The theoretical foundations of CSCW and CSCL are largely influenced by Vygotsky [30], who stressed that learning and other higher psychological processes originally take place socially, intersubjectively. Piaget, too, pointed to inter-subject processes like conflicting perspectives as a fundamental driver for creativity and cognitive development. Despite this powerful insight, Vygotsky, Piaget and their followers generally maintain a psychological focus on the individual mind in their empirical studies and do not systematically investigate the intersubjective phenomena of small-group interaction.

A science of group interaction would aim to unpack what happens at the small-group unit of analysis. Thus, it would be particularly relevant for $\mathrm{CSCW}$ and CSCL, even if it may not be as directly applicable to other forms of working or learning, where the individual or the community level predominates. As a science of the group, it would complement existing theories of working, learning and cognition, to the extent that they focus either on the individual or the community or that they reduce group phenomena to these other levels of description.

In the chapters of Studying Virtual Math Teams [28] and of Group Cognition [26], my colleagues and I have reviewed some of the research literature on small-group learning, on small-group processes and on collaborative mathematics. We have observed that traditional "small-group studies" generally look for quantitative correlations among variables - such as the effect of group size on measures of participation-rather than trying to analyze group knowledge-building processes directly. Studies of small-group processes from psychology, sociology and other 
social sciences also tend to focus on non-cognitive aspects of group process or else attribute all cognition to the individual minds rather than to group processes. There are some notable exceptions; in particular, we viewed $[2 ; 4 ; 22 ; 23]$ as important preliminary studies - although even they tend to "black box" the group processes.

Even theories that seem quite relevant to our concerns, like distributed cognition [11], actor-network theory [14], situated cognition [15], ethnomethodology [8] and activity theory adopt a different focus, generally on interaction of individuals with artifacts rather than among people. In particular, recent commentaries on situated cognition [18] and distributed cognition [1] frame the issues at the individual level, even reducing all cognitive phenomena to neural phenomena. At the other extreme, social theories focus on community phenomena like division of labor, apprenticeship training, linguistic structure, laboratory organization. For all its insight into small group interaction and its analysis, ethnomethodology maintains a sociological perspective. Similarly, even when activity theory addresses the study of teams - in the most detail in Chapter 6 of [5] - it is mostly concerned with the group's situation in the larger industrial and historic context; rather than analyzing how groups interactionally build knowledge, it paraphrases how they deal politically with organizational management issues. These theories provide valuable insights into group interaction, but none of them thematizes the small-group level as a domain of scientific study. As sciences, these are methodologically and conceptually at root sciences of the individual or of the society, not of the collaborative group.

Each of the three levels of description is populated with a different set of phenomena and processes. For instance, in online chat individuals interpret recent postings and design new postings in response, the group constructs, maintains and repairs a joint problem space and the community evolves its shared methods of social organization. The description of the individual level is the province of psychology; that of the community is the realm of sociology or anthropology; the small-group level has no corresponding science.

A science of group interaction would take its irreducible position between the psychological sciences of the individual and the social sciences of the community-much as biology analyzes phenomena that are influenced by both chemicals and organisms without being reducible to either. The science of group interaction would fill a lacuna in the multi-disciplinary work of the human sciences. This science would not be primarily oriented toward the "low level" processes of groups, such as mechanical or rote behaviors, but would be concerned with the accomplishment of creative intellectual tasks. Intellectual teamwork, knowledge work and knowledge-building activities would be prototypical objects of study. The focus would be on group cognition.

The bifurcation of the human sciences into individual and societal creates an irreconcilable opposition between individual creative freedom and restrictive social institutions. A science of group cognition would flesh out the concept of structuration, demonstrating with detailed analyses of empirical data how group interactions can mediate between individual behavior and social practices [28, ch. 11].

The term group cognition does not signify an object or phenomenon to analyze like brain functions or social institutions.
It is a proposal for a new science or focus within the human sciences. It hypothesizes:

When small groups engage in cooperative problem solving or collaborative knowledge building, there are distinctive processes of interest at the individual, small-group and community levels of analysis, which interact strongly with each other. The science of group cognition is the study of the processes at the small-group level.

The science of group cognition is a human science, not a predictive science like chemistry nor a predominantly quantitative one like physics. It deals with human meanings in unique situations, necessarily relying upon interpretive case studies and descriptions of inter-personal processes.

Processes at the small-group level are not necessarily reducible to processes of individual minds, not do they imply the existence of some sort of group mind. Rather, they generally take place through the weaving of semantic and indexical references within a group discourse. The indexical field [9] or joint problem space [29] co-constructed through the sequential interaction of a group [3] has the requisite complexity to constitute an irreducible cognitive act in its own right. Cognitive science broadened the definition of "cognition" beyond an activity of human minds in order to include artificial intelligence of computers. What counts as cognitive is now a matter of computational complexity. Anything that can compute well enough to play chess or prove theorems can be a cognitive agent-whether they are a person, computer or collaborative small group [25].

A science of group cognition is timely and relevant, as indicated by the rise of the CSCW and CSCL fields. The $21^{\text {st }}$ century will increasingly rely on small groups - due to networked computers creating new means of group intellectual production, with the power to overcome the limitations of the individual mind. The dominance of the individual in production and in science was part of the larger epochal trend of industrialization. Now forces of instantaneous communication, globalization and ecological crisis seem to be bringing about a transformation of that historic trend, resulting in the rising prominence of the small group as an important mediator between the isolated individual and an increasingly abstract society. The small group is becoming an effective new form in the social relations of intellectual production.

Having motivated the development of a science of group cognition as future work, let us see how the Virtual Math Teams (VMT) Project [28] may have begun to prepare the way. We start with how the VMT world of online collaboration was constructed as an object of study.

\section{DESIGNING A TESTBED FOR GROUP INTERACTION}

The first step in our design-based research process was to start simply and see what issues came up. We had seen in face-to-face case studies that there were problems with (a) recording and transcribing the verbal interaction, (b) capturing the visual interaction and (c) knowing about all the influences on the interaction. We decided to form groups of students who did not know each other and who only interacted through text chat. Students were recruited through the Math Forum at Drexel University, an established online resource center. We used AIM, AOL's Instant Messaging system, which was freely available and 
was already familiar to many students. We included a researcher in the chat room with each small group of students. The facilitator told the students their math task, dealt with any technical difficulties, posted drawings from the students on a web page where they could be seen by all the students, notified the group when the session was over and saved an automatically generated $\log$ of the chat. In this way, we obtained a complete and objective $\log$ of the interaction, captured everything that the students shared on their computers and excluded any unknown influences from affecting the interaction.

The issue of including everything affecting the interaction is a subtle issue. Of course, the interaction is influenced by the life histories, personalities, previous knowledge and physical environment of each student. A student may have windows other than AIM open on the computer, including Internet browsers with math resources. A student may be working out math problems on a piece of paper next to the computer. Or a student may leave the computer for some time to eat, listen to music, talk on the phone and so on, without telling anyone in the chat. So we do not have information about everything involved in a particular student's online experience. We do not even know the student's gender or age. We do not know if the student is shy or attractive, speaks with an accent or stutters. We do not know if the student usually gets good grades or likes math. We do not know what the student is thinking or feeling. We only know that the students are in an approximate age group and academic level — because we recruited them through teachers. However, the VMT Project is only concerned with analyzing the interaction at the group unit of analysis. Notice that the things that are unknown to us as researchers are also unknown to the student group as a whole. The students do not know specifics about each other's background or activities - except to the extent that these specifics are brought into the chat. If they are mentioned or referenced in the chat, then we can be aware of them the same as are the students.
In addition to personal influences, the chat is responsive to linguistic and cultural matters. Of course, both students and researchers must know English to understand the chats. In particular, forms of English that have evolved with cell-phone texting have introduced abbreviations, symbols and emoticons into the chat language. The linguistic subculture of teenagers also shows up in the VMT chats. An interdisciplinary team of researchers comes in handy for interpreting the chats. In our case, the research team brought in experience with online youth lingo based on their backgrounds as Math Forum staff, teachers or parents.

The early AIM chats used simple math problems, taken from standardized math tests and Math Forum Problems-of-the-Week. One experiment to compare individual and group work used problems from a standardized multiple-choice college-admissions test. These problems had unique correct answers. While these provided a good starting point for our research, they were not well suited for collaborative knowledge building. Discourse around them was often confined to seeing who thought they knew the answer and then checking for correctness. For the VMT Spring Fests in 2005, 2006 and 2007, we moved to more involved math topics that could inspire several hours of joint inquiry.

Even with straightforward geometry problems, it became clear that students needed the ability to create, share and modify drawings within the VMT environment. We determined that we needed an object-oriented draw program, where geometric objects could be manipulated (unlike a pixel-based paint program). We contracted with the developers of ConcertChat to use and extend their text chat and shared whiteboard system, which is now available in Open Source. This system included a graphical referencing tool as well as social awareness and history features [17]. (See Figure 1.) In order to help students find desirable chat rooms and to preserve team findings for all to see, we developed the VMT Lobby and integrated a Wiki with the Lobby and chat

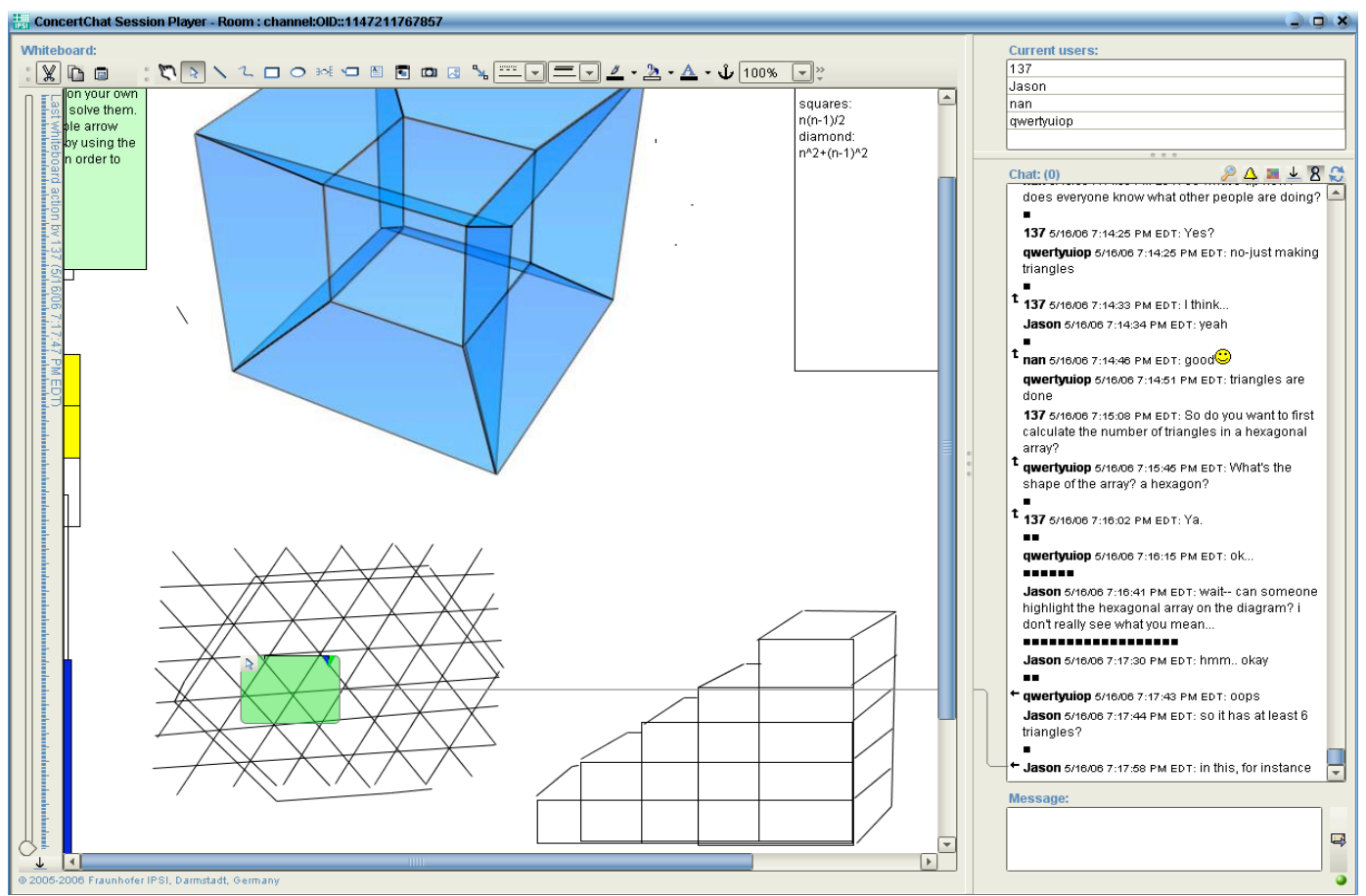

Figure 1. The VMT chat room with whiteboard. 
rooms. Gradually, the technology and the math topics became much more complicated in response to the needs that were revealed when we analyzed the trials of the earlier versions of the VMT service. As the system matured, other research groups began to use it for their own trials, with their own math topics, procedures, analytic methods or even new technical features. These groups included researchers from Singapore, Rutgers, Hawai' i, Romania and Carnegie-Mellon [28].

The evidence for the adequacy of a testbed for design-based research lies in the success of the analyses to reveal how the prototyped environment is working at each design iteration and to provide ideas based on problems encountered by users to drive the design further. Therefore, we now turn to the analyses of actual interactions within the virtual math teams to see if the testbed produced adequate data for understanding group cognition in this context.

\section{ANALYZING GROUP INTERACTION}

The approach to chat interaction analysis that emerged in the VMT Project will be discussed in this section in terms of a number of issues (which correspond to general issues of research methodologies, as indicated in parentheses):

\subsection{Group Cognition in a Virtual Math Team (Research Question)}

Learning - whether in a classroom, a workplace or a research lab - is not a simplistic memorization or storage of facts or propositions, as traditional folk theories had it. The term learning is a gloss for a broad range of phenomena, including: the development of tacit skills, the ability to see things differently, access to resources for problem solving, the discursive facility to articulate in a new vocabulary, the power to explain, being able to produce arguments or the making of new connections among prior understandings. We can distinguish these phenomena as taking place within individual minds, small-group interactions or community practices. The analysis of learning phenomena at these various levels of analysis requires different research methodologies, appropriate to corresponding research questions. The VMT Project was intended to explore the phenomena of group cognition and accordingly pursued the research question:

How does learning take place in small groups, specifically in small groups of students discussing math in a text-based online environment? What are the distinctive mechanisms or processes that take place at the small-group level of description when the group is engaged in problem-solving or knowledge-building tasks?

While learning phenomena at the other levels of analysis are important and interact strongly with the group level, we have tried to isolate and make visible the small-group phenomena and to generate a corpus of data for which the analysis of the group-level interactions can be distinguished from the effects of the individual and community levels.

The methods used to gather and analyze one's data should be appropriate to one's research question. To support such research, one must generate and collect data that are adequate for the selected kinds of analysis. Because we were interested in the group processes that take place in virtual math teams, we had to form teams that could meet together online. In the Spring Fests, students had to be able to come back together in the same teams on several subsequent occasions. The VMT environment had to be instrumented to record all messages and activities that were visible to the whole team in a way that could be played back by the analysts. The math problems and the feedback to the teams had to be designed to encourage the kinds of math discussions that would demonstrate processes of group cognition, such as formulating questions and proposals, coordinating drawings and textual narratives, checking proposed symbolic solutions, reviewing the team's work and so on. A sense of these desirable group activities and the skill of designing problems to encourage them had to develop gradually through the design-based research iterations.

\subsection{Non-laboratory Experimental Design (Validity)}

Of course, to isolate the small-group phenomena we do not literally isolate our subject groups from individuals and communities. The groups consist of students, who are individuals and who make individual contributions to the group discourse based on their individual readings of the discourse. In addition, the groups exist and operate within community and social contexts, drawing upon the language and practices of their math courses and of their school, teen and online subcultures. These are essential features of a real-world context and we would not wish to exclude them even to the extent possible by confining the interaction to a controlled laboratory setting. We want the students to feel that they are in a natural setting, interacting with peers. We do not try to restrict their use of language in any way (e.g., by providing standardized prompts for chat postings or by forced scripting of their interactions with each other).

We are designing a service that can be used by students and others under a broad array of scenarios: integrated with school class work, as extra-curricular activities, as social experiences for home-schooled students, as cross-national team adventures or simply as opportunities (in a largely math-phobic world) to discuss mathematics. To get a sense of how such activities might work, we have to explore interactions in naturalistic settings, where the students feel like they are engaged in such activities rather than being laboratory subjects.

\subsection{Data Collection at the Group Level of Description (Unit of Analysis)}

Take the network of references in a chat-threading diagram as an image of meaning making at the group level. One could almost say that the figure consists entirely of contributions from individuals (the chat postings and whiteboard drawings) and resources from the math community; that everything exists on either the individual or community level, not on the group level. Yet, what is important in the figure is the network of densely interwoven references, more than the objects that are connected by them. This network exists at the group level. It mediates the individual and the community by forming the joint problem space [21;29], indexical ground [9], referential network or situation within which meanings, significant objects and temporal relations are intersubjectively co-constructed. On the individual level, these shared group meanings are interpreted and influence the articulation of subsequent postings and actions. On the community level, the meanings may contribute to a continually evolving culture through structuration processes. The VMT Project is oriented toward the processes at the group unit of analysis, which build upon, connect and mediate the individual and community phenomena. 
Elements from the individual and community levels only affect the group level if they are referenced in the team's interaction. Therefore, we do not need to gather data about the students or their communities other than what appears in the interaction record. We do not engage in surveys or interviews of the students or their teachers. For one thing, the design of the VMT Project prohibits access to these sources of data, because the students are only available online during the chat sessions. External sources of data would be of great interest for other research questions having to do with individual learning or cultural changes, but for our research question, they are unnecessary and might even form a distraction or skew our analysis because it would cause our readings of the postings to be influenced by information that the group had not had.

By moving to the disembodied online realm of group cognition in virtual math teams, it is easier for us to abandon the positivist metaphors of the mechanistic worldview. Not only is it clear that the virtual group does not exist in the form of a physical object with a persistent memory akin to a computer storage unit, but even the individual participants lack physical presence. All that exists when we observe the replayed chats are the traces of a discourse that took place years ago. Metaphors that might come naturally to an observer of live teamwork in a workplace or classroom-personalities, the group, learning, etc.-no longer seem fundamental. What exist immediately are the textual, graphical and symbolic inscriptions. These are significant fragments, whose meaning derives from the multi-layered references to each other and to the events, artifacts and agents of concern in the group discourse. This meaning is as fresh now as when the discourse originated, and can still be read off the traces by an analyst, much as by the original participants. This shows that the meanings shared by the groups are not dependent upon mental states of the individual students - although the students may have had interpretations of those meanings in mind, external to the shared experience. The form of our data reinforces our focus on the level of the shared-group-meaning making as an interactional phenomenon rather than a psychological one.

\subsection{Instrumentation and Data Formats (Objectivity)}

It was noted above that when one videotapes small-group interactions a number of practical problems arise. Data on face-toface classroom collaboration runs into issues of (a) recording and transcribing the verbal interaction, (b) capturing the visual interaction and (c) knowing about all the influences on the interaction. The data is in effect already partially interpreted by selective placement of the microphone and camera. It is further interpreted by transcription of the talk and is restricted by limited access to facial expressions and bodily gestures. Much happens in a classroom influencing the student teams, which is not recorded.

The online setting of the VMT sessions eliminates many of these problems. As already described, the automatic computer log of the session captures everything that influences the group as a whole. This includes all the postings and whiteboard activity, along with their precise timing. They are captured at the same granularity as they are presented to the students. Chat postings appear as complete messages, defined by the author pressing the Enter button. Whiteboard textboxes appear as complete, when the author clicks outside of the textbox. Whiteboard graphics appear gradually, as the author positions each graphical element. Computer-generated social-awareness messages (when people enter or exit the chat room, begin or end typing, move a graphical object, etc.) are also accurately recorded. The precision of the log recording is assured because it consists of the original actions (as implemented by the computer software) with their timestamps. The original display to the students is generated from the same data that is viewed by analysts.

For our analysis of chats, we use a VMT Replayer. The Replayer is simply an extended version of the Java applet that serves as the chat/whiteboard room in the VMT environment. The reproduced chat room is separated by a thin line at the bottom from a VCRlike interface for replaying the session. The session can be replayed in real time or at any integral multiple of this speed. It can be started and stopped at any point. An analyst can drag the pointer along the timeline to scroll both the whiteboard history and the chat history in coordination. One can also step through the recorded actions, including all the awareness messages. In addition, spreadsheet logs can be automatically generated in various useful formats.

The data analyzed in the VMT Project is recorded with complete objectivity. There is no selectivity involved in the data generation, recording or collecting process. Furthermore, the complete recording can be made available to other researchers as a basis for their reviews of our analyses or the conducting of their own analyses. For instance, there have been multiple published analyses of the VMT data by other research groups following somewhat different research questions, theories and methods. While VMT collaborative sessions are each unique and in principle impossible to reproduce, it is quite possible to reproduce the unfolding of a given session from the persistent, comprehensive and replayable record.

\subsection{Collaborative Data Sessions (Reliability)}

Interpretation of data in the VMT Project first begins with an attempt to describe what is happening in a chat session. We usually start this process with a data session [12] involving six to twelve researchers. A typical data session is initiated by a researcher who is interested in having a particular segment of a session log discussed by the group. Generally, the segment seems to be both confusing and interesting in terms of a particular research question.

For our data sessions, we sit around a circle of tables and project an image of the VMT Replayer onto a screen visible to everyone. Most of us have laptop computers displaying the same Replayer, so that we can scan back and forth in the segment privately to explore details of the interaction that we may want to bring to the attention of the group. The group might start by playing the segment once or twice in real time to get a feel for how it unfolds. Then we typically go back to the beginning and discuss each line of the chat sequentially in some detail.

The interpretation of a given chat line becomes a deeply collaborative process. Generally, one person will make a first stab at proposing a hypothesis about the interactional work that line is doing in the logged discourse. Others will respond with suggested refinements or alternatives to the proposal. The group may then engage in exploration of the timing of chat posts, references back to previous postings or events. Eventually the data analysis will move on to consider how the student group took up the posting. An interesting interpretation may require the analysts to return to earlier ground and revise previous understandings. 
It is usually first necessary to clarify the referential structure of the chat postings and how the postings relate to events in the whiteboard or to the comings and goings of participants. The threading of the chat postings provides the primary structure of the online, text-based discourse in much the same way that turn taking provides the core structure of spoken informal conversation. Because of the overlap in the typing of chat postings, it is sometimes tricky to figure out who is responding to what. Looking at the timestamps of posts and even at the timestamps of awareness messages about who is typing can provide evidence about what was visible when a posting was being typed. This can often suggest that a given post could or could not have been responding to a specific other post, although this is sometimes impossible to determine. When it is hard for the analyst to know the threading, it may have also been hard for most of the chat participants (other than the typist) to know; this may result in signs of trouble or misunderstandings in the subsequent chat.

Since our research question involves the group as the unit of analysis, we do not raise questions in the data session about what one student or another may have been doing, thinking or feeling as an individual. Rather, we ask what a given posting is doing interactionally within the group process, how it responds to and takes up other posts and what opportunities it opens for future posts. We look at how a post is situated in the sequential structure of the group discourse, in the evolving social order and in the team's meaning making. What is this posting doing here and now in the referential network? Why is it "designed to be read" [16] in just this way? How else could it have been phrased and why would that not have achieved the same effect in the group discourse?

We also look at how a given posting positions [10] both the author and the readers in certain ways. We do not attribute constant personalities or fixed roles to the individuals, but rather look at how the group is organized through the details of the discourse. Perhaps directing a question toward another student will temporarily bestow upon her a form of situated expertise [32] such that she is expected to provide an extended sequence of expository postings.

In coding analysis, it is conventional to train two people to code some of the same log units and to compare their results to produce an inter-rater reliability measure. In our chat interaction analysis, we do not pretend that the $\log$ can be unproblematically partitioned into distinct units, which can be uniquely assigned to a small number of unambiguous codes. Rather, most interesting group discourse segments have a complex network of interwoven references. The analysis of such log segments requires a sophisticated human understanding of semantics, interpersonal dynamics, mathematics, argumentation and so on. Much is ultimately ambiguous and can be comprehended in multiple ways-sometimes the chat participants were intentionally ambiguous. At the same time, it is quite possible for analysts to make mistakes and to propose analyses that can be shown to be in error. To ensure a reasonable level of reliability of our analyses, we make heavy use of data sessions. This ensures that a number of experienced researchers agree on the analyses that emerge from the data sessions. In addition, we try to provide logs - or even the entire session data with the Replayer-in our papers so that readers of our analyses can judge for themselves the interpretations that are necessarily part of chat analysis.

\subsection{Describing Social Practices (Generalizability)}

The research question that drives the VMT Project is: What are the distinctive mechanisms or processes that take place at the small-group level of description when the group is engaged in problem-solving or knowledge-building tasks? Therefore, we are interested in describing the inter-personal practices of the groups that interact in the VMT environment.

Vygotsky [30] argued that learning takes place inter-subjectively (in dyads or groups) before it takes place intra-subjectively (by individuals). For instance, in his analysis of an infant and mother (p. 56), he outlines the process through which the infant's unsuccessful grasping at some object becomes established by the mother-child dyad as a pointing at the object. This shared practice of pointing subsequently becomes ritualized by the dyad and then mediated and "internalized" by the infant as a pointing gesture. The pointing gesture - as a foundational form of deictic reference - is a skill of the young child, which he can use for selecting objects in his world and learning about them. His mother understands the gesture because it was intersubjectively established with her. In this prototypical example, Vygotsky describes learning as an inter-subjective or small-group practice of a dyad.

While we can imagine that Vygotsky's description is based on a concrete interaction of a specific infant and mother in a particular time and place, the pointing gesture that he analyzed is ubiquitous in human culture. In this sense, the analysis of a unique interaction can provide a generalizable finding. The science of ethnomethodology (the study of the methods used by people) [8] is based on the fact that people in a given culture or linguistic community share a vast repertoire of social practices for accomplishing their mundane tasks. It is only because we share and understand this stock of practices that we can so quickly interpret each other's verbal and gestural actions, even in novel variations under unfamiliar circumstances. The analysis of unique case studies can result in the description of social practices that are generalizable. The methods developed in specific situated encounters are likely to be typical of a broad range of cases under similar conditions.

By describing the structure of group practices in detailed case studies, we can characterize general methods of group behavior, group learning or group cognition. Findings from analyses of case studies can lead to the proposal of theoretical categories, conceptualizations, structures or principles - in short, to a science of group interaction.

\section{CONCEPTUALIZING GROUP INTERACTION}

As discussed above, students in virtual math teams are active as individuals, as group participants and as community members. They each engage in their own, private individual activities, such as reading, interpreting, reflecting upon and typing chat messages. Their typed messages also function as group actions, contributing to the on-going problem solving of the team. Viewed as community events, the chats participate in the socialization process of the society, through which the students become increasingly skilled members of the community of mathematically literate citizens.

A thesis of the theory of group cognition is, "Small groups are the engines of knowledge building. The knowing that groups build up 
in manifold forms is what becomes internalized by their members as individual learning and externalized in their communities as certifiable knowledge" [26, p. 16]. Despite their centrality, small groups have not been theorized or studied extensively.

Some small-group literature has been produced from either the methodological perspective of psychology or that of sociology, primarily since World War II. Traumatized by the mass-culture horrors of fascism and by extreme forms of mentalist pseudoscience, these predominantly behaviorist studies focused on the negative aspects of "group think" and caricatured the notion of "group mind"-which had a well-respected history before the rise of positivism [31].

More recent theories like distributed cognition, situated action or activity theory actually conduct case studies of small-group interaction, but they do not theorize the small group as their unit of analysis and therefore they do not produce descriptions of small-group practices as such. Even Hutchins [11], in studying distributed cognition in the wild, does not thematize the interpersonal interactions, but focuses on the cognitive unit of analysis, simply broadening it to include the external computational and physical representational artifacts that an individual worker uses. Furthermore, the cognitive accomplishments he studies are routine, well scripted procedures that do not involve creative solutions to ill-structured problems; the coordination of the navigational team is fixed by naval protocol, not co-constructed through the interaction.

The VMT studies provide a model for describing the small-group practices as distinct from individual and community processes. They look at rich interactions in groups larger than dyads, where individual identities play a smaller role. They analyze group efforts in high-order cognition such as mathematical problem solving and reflection on their problem-solving trajectory. They investigate groups that meet exclusively online, where the familiar visual, physical and aural modes of communication are unavailable, and where communication is mediated by designed technological environments. A number of findings become prominent in these analyses. We shall review two findings here. (a) One is that much group work is sustained and driven forward by proposals and responses to them. (b) Another is that group interactions form a social order, which can often be characterized in terms of a temporal dimension, a joint problem space and an interaction space.

\subsection{Proposal-driven Sustained Group Activity}

Careful review of many VMT logs shows that group interaction in these sessions is driven forward and sustained by various kinds of proposals. One of the first findings of the VMT Project was the role of "math proposal adjacency pairs" [26, ch. 21 esp. pp. 442456]. These are simply a form of proposal adjacency pairs as found in informal face-to-face conversation, except that they deal with mathematical matters and they are only "adjacent" once their timing has been adjusted for threading. Technically, they might better be termed "math proposal response pairs," except that the term "adjacency pair" brings in the valuable theoretical connotations from conversation analysis [20].

A proposal is not a solitary speech act. It involves minimally two acts: a bid and a response. For instance, a question is only gradually formulated. People respond to an original opening bid and thereby define the question as an activity taken up in a certain way by the group [32].
Through the proposal co-construction process, the group work becomes "object-oriented." The group orients to some mathematical object. Early in a session, the object may be based on a phrase from the task set for the group by the organizers of the VMT session. Later, the group members may explicate it in terms of visual representations or graphical objects in the whiteboard or symbolic math expressions in the chat.

The idea that group activity is strongly "object-oriented" is an important principle of activity theory $[6 ; 13]$. It stresses the taskdriven nature of group work. In the occupational settings that activity theory generally studies, activities often aim to accomplish a goal that has been established in advance (e.g., by management) as the purpose of the group. By highlighting the role of proposals as important means of structuring group interaction, the VMT studies of learning settings reveal an interactional mechanism through which groups co-construct their own work goals in concrete detail.

Student groups in VMT sessions are highly responsive to the tasks that are pre-defined before they enter the chat room. These tasks are stated for them in various ways - on special web pages and/or by the moderator in chat - and the students clearly orient to them. However, one of the first things that the student group does is to discuss the task they will pursue. This is often put in the form of a posting like, "OK, let's figure out...." This is a proposal for what the group should work on next. It is selective of some feature of a broader task that was given to the group. As a proposal, it elicits a response from the rest of the group. The response further develops the proposed task. By highlighting the structure of the proposal, the analyses of the VMT Project show how the group itself accomplishes object orientation as an interactional achievement of the group. The object of a group's work is not given in advance and fixed for all time. Nor is it defined only at the level of a goal for the whole session. It is worked out and continually refined by the group interaction, even if it references texts and motivations from outside the group discourse. Furthermore, objects that orient the group work are proposed for small sequences of interaction as well as for the session-long sequences, as each new proposal is taken up.

The proposal structure introduces a temporal structure. A proposal often puts forward a task for the group to take on in the (near) future, possibly as a next step in its work. Sometimes-like at the end of a session that will be followed by another session of the same team - a proposal will plan for a future session. By its nature, a proposal bid creates possible next actions for the group, such as accepting, rejecting, questioning or ignoring the bid. In turn, the second part of the math proposal pair references back to the first part, which by now exists in the interaction past. It may well also reference events further back in the team's past, such as work already done or decisions previously made. The proposal as a whole, as it unfolds over potentially many actions, is always situated firmly in the present network of references. Thus, the proposal process contributes to establishing the temporal dimension of the group's work, with references to future, past and present events.

It is common to make diagrams of the proposal-response structure of chats. Such representations can be an important part of a science. The response structure, uptakes, adjacency pairs, sequences, etc. are central to an analysis of a chat interaction. This theme is familiar in the broader literature on chat. The diversity of representations proposed (each with their rationale) indicates that this is a problematic issue as well as an important one for a future 
science of group cognition. Similarly, many researchers try to develop and apply coding schemes to analyze chats. A science of group cognition will have to take a stand on coding and on the appropriateness of specific coding schemes to interaction analysis.

The temptation to develop automated software [7; 19] to construct graphical representations of the response structure and to categorize utterances may ironically serve to highlight the issues involved in making simplistic assumptions about the objective nature of the response structure and of the utterance character. A threading or uptake graph may make it look like postings exist with measurable attributes and fixed relationships, like the objects of Newtonian mechanics, with their precise location, mass and velocity. However, chat messages are more analogous to quantum particles, with their indeterministic and probabilistic characteristics. Whether a posting is a math proposal, a question or a joke depends on how an interpretive, thread-producing "reading" of it not only construes its uptake by subsequent postings, but also how it situates that posting in relation to previous postings. A particular posting may reference past and current artifacts, events and agents, but it also projects relevant "nexts," responses or uptakes by opening a field of possibilities. This is more complicated and less well defined than implied by a static diagram of nodes and links, however useful such a diagram may be to support visual reasoning about specific issues involving the flow of a chat. It may make more sense to treat postings as mediating agents in Latour's [14] sense, as an alternative to metaphors from mechanistic theories of causation.

Proposal structures in VMT data can be more complicated than traditional analyses of adjacency pairs in studies of talk-ininteraction. Most case studies inspired by conversation analysis look at short sequences like a single adjacency pair or a pair that is temporarily interrupted by clarifications or repairs. The VMT Spring Fests allow analysis of longer sequences. In these, one sees mechanisms by means of which the work of a group is integrated into a layered temporal unity. The study of proposal mechanisms may lead to the identification of social structure in groups.

\subsection{The Social Order of Group Interaction}

Temporal structure is one dimension of the social order that a collaborative small group co-constructs of, by and for its interaction. Proposals are but one interactive mechanism for establishing the social order that supports the achievement of group cognition. By looking at bridging methods in longer sequences and across temporal and other discontinuities, analyses of VMT chats [21] demonstrate the importance of the temporal dimension in addition to the content and relational dimensions that had been proposed by previous related research. This suggests three dimensions to the social order established by virtual math teams and other small groups engaged in group cognition:

- The temporal dimension of ordered events.

- The problem space of shared knowledge artifacts.

- The interaction space of positioned actors.

The first dimension of social order, the temporal dimension, was just discussed in terms of the ways in which proposal interactions are themselves temporally structured, with references to possible next responses, past resources and the current situation. The temporal dimension is also woven as part of the referential network of meaning that is built up through the group discourse. In particular, temporal indexicals (like then) and verb tenses establish the indexical ground of deictic reference [9], which is part of the shared meaning structure that makes sense of references to events and locates them within their temporal ordering.

In discourses about math, the second dimension, the problem space, is traditionally conceived of within the cognitivist tradition as a mental representation of mathematical relationships. The analysis of the work of virtual math teams [3] shows that the group works out a shared notion of the math object, for instance by constructing visualizations in the whiteboard and instructing the group members to see them in a certain way. There is often a coordinated movement back and forth between visual, narrative and symbolic reasoning that gradually objectifies the math object into a rich, interconnected, meaningful multiplicity of significances and realizations. The representation of the object for the group does not lie hidden in individual minds like the data structure of an artificial intelligence software system. It consists of a network of visible inscriptions in the visual interface of the VMT environment, tied together into a meaningful whole by the set of carefully crafted references within the group interaction. The object exists as an artifact, a physical object that is meaningful [26, ch. 16]. However, in the case of math objects that are the result of extensive group work, there is not a single identifiable artifact; the math object consists of a "tree of multiple realizations" [24; 27] united by the group discourse and only imperfectly objectified in a single phrase or symbol.

In particular, once the rich experience of the group interaction that built the math artifact is summarized or sedimented into a single sign and passed on to others who were not involved in the original experience (e.g., late-comers or newcomers), the full meaning of the artifact is hard to come by. This is the problem of math education. For new individuals to build anything like a mental representation of a math artifact, they need to go through a process like that which Vygotsky termed internalization. Either they need to experience a group process like those that occur in virtual math teams or they need to simulate such a process on their own. One often sees math students sketching visual reasoning diagrams on paper, playing around with symbolisms and arguing with themselves as though they were acting out the parts of a complete team. The path to math comprehension seems to require the practices of group problem solving, which experienced experts have learned to individuate and to conduct as individuals, imagining the visualizations and speaking the discourse subvocally.

The third dimension of social order is the interaction space of intersubjective relations. We characterize this in terms of positioning [10]. In the VMT environment, there is no power hierarchy or other system of roles among the students. (The adult mentor who may be in the chat room with the students is, of course, an authority figure, but tends to play a minimal role in the session and rarely enters into the math work or interactions among the team. The mentor is positioned as being outside of the team; often by the mentor's own postings.) Researchers often discuss collaboration in terms of roles. Some even advocate scripting or assigning fixed roles to students to make sure that certain functions of group process are carried out - such as leading the discussion, watching the time allotted for the session, summarizing the group accomplishments, monitoring the active participation of all members, controlling turn taking. In contrast to such an imposed approach, an analysis in terms of positioning views roles as fluidly changing, based on details of the group discourse. 
Perhaps the clearest example of positioning arises in questioning. When one person asks another what some term means or how a result was derived, the questioner may be positioned as lacking knowledge and the addressee as having situated expertise. What this means is that the first person cedes the second the floor. The questioner will refrain from posting anything for a while and will expect the other group members to do likewise while the second person - the temporary expert for purposes of this question-will be expected to post a series of expository messages responding to the question. Questions are carefully designed to engage in positioning moves and other interpersonal work. Through methods like questioning and displays of individual knowledge, group members co-construct the intersubjective fabric of the group, often starting from a condition where there are no differentiations.

\subsection{The Analysis of Group Interaction}

In this paper, we have only been able to touch on a couple examples of our preliminary contributions to a science of group interaction. Additional findings from the VMT Project relevant to a new science of group interaction are available in $[3,21,26,28$ and 32]. Of course, these tentative explorations do not constitute a mature science; the trust of this paper is to call for an interdisciplinary effort within the CSCL and CSCW research community. The VMT Project is intended to illustrate some aspects of a possible approach for such a science. The point is to understand better the unique and powerful mechanisms that occur at the group level of description within effective knowledge building efforts of small groups.

\section{REFERENCES}

[1] Adams, F. \& Aizawa, K. (2008) The bounds of cognition, Blackwell, Malden, MA

[2] Barron, B. (2003) When smart groups fail, The Journal of the Learning Sciences, 12 (3), pp. 307-359.

[3] Çakir, M. P., Zemel, A., \& Stahl, G. (2009) The joint organization of interaction within a multimodal CSCL medium, International Journal of Computer-Supported Collaborative Learning (ijCSCL), 4 (2).

[4] Cohen, E. G., Lotan, R. A., Abram, P. L., Scarloss, B. A., \& Schultz, S. E. (2002) Can groups learn?, Teachers College Record, 104 (6), pp. 1045-1068.

[5] Engeström, Y. (2008) From teams to knots, Cambridge University Press, Cambridge, UK.

[6] Engeström, Y. \& Toiviainen, H. (2009) Co-configurational design of learning instrumentalities: An activity-theoretical perspective. In S. Ludvigsen, A. Lund, \& R. Säljö (Eds.), Learning in social practices. ICT and new artifacts: Transformation of social and cultural practices, Pergamon.

[7] Erkens, G. \& Janssen, J. (2008) Automatic coding of communication in collaboration protocols, International Journal of Computer-Supported Collaborative Learning (ijCSCL), 3 (4).

[8] Garfinkel, H. (1967) Studies in ethnomethodology, PrenticeHall, Englewood Cliffs, NJ.

[9] Hanks, W. (1992) The indexical ground of deictic reference. In C. Goodwin \& A. Duranti (Eds.), Rethinking context: Language as an interactive phenomenon, Cambridge University Press, Cambridge, UK.
[10] Harré, R. \& Moghaddam, F. (2003) Introduction: The self and others in traditional psychology and in positioning theory. In R. Harré \& F. Moghaddam (Eds.), The self and others, Praeger, Westport, CT, pp. 1- 11.

[11] Hutchins, E. (1996) Cognition in the wild, MIT Press, Cambridge, MA.

[12] Jordan, B. \& Henderson, A. (1995) Interaction analysis: Foundations and practice, Journal of the Learning Sciences, 4 (1), pp. 39-103.

[13] Kaptelinin, V. \& Nardi, B. (2006) Acting with technology: Activity theory and interaction design, MIT Press, Cambridge, MA.

[14] Latour, B. (2007) Reassembling the social: An introduction to actor-network-theory, Cambridge University Press, Cambridge, UK.

[15] Lave, J. \& Wenger, E. (1991) Situated learning: Legitimate peripheral participation, Cambridge University Press, Cambridge, UK.

[16] Livingston, E. (1995) An anthropology of reading, Indiana University Press, Bloomington: IN.

[17] Mühlpfordt, M. \& Stahl, G. (2007) The integration of synchronous communication across dual interaction spaces, In: Proceedings of international conference on Computer Support for Collaborative Learning (CSCL 2007), New Brunswick, NJ.

[18] Robbins, P. \& Aydede, M. (Eds.) (2009) The Cambridge handbook of situated cognition, Cambridge University Press, Cambridge, UK.

[19] Rosé, C., Wang, Y.-C., Cui, Y., Arguello, J., Stegmann, K., Weinberger, A., \& Fischer, F. (2008) Analyzing collaborative learning processes automatically: Exploiting the advances of computational linguistics in CSCL, International Journal of Computer-Supported Collaborative Learning (ijCSCL), 3 (3), pp. 237-272.

[20] Sacks, H., Schegloff, E. A., \& Jefferson, G. (1974) A simplest systematics for the organization of turn-taking for conversation, Language, 50 (4), pp. 696-735.

[21] Sarmiento, J. (2007) Bridging: Interactional mechanisms used by online groups to sustain knowledge building over time, In: Proceedings of international conference on Computer-Supported Collaborative Learning (CSCL '07), New Brunswick, NJ.

[22] Sawyer, R. K. (2003) Group creativity: Music, theater, collaboration, Lawrence Erlbaum, Mahwah, NJ.

[23] Schwartz, D. (1995) The emergence of abstract representations in dyad problem solving, Journal of the Learning Sciences, 4 (3), pp. 321-354.

[24] Sfard, A. (2008) Thinking as communicating: Human development, the growth of discourses and mathematizing, Cambridge University Press, Cambridge, UK.

[25] Stahl, G. (2005) Group cognition: The collaborative locus of agency in CSCL, In: Proceedings of international conference on Computer Support for Collaborative Learning (CSCL '05), Taipei, Taiwan, pp. 632-640. 
[26] Stahl, G. (2006) Group cognition: Computer support for building collaborative knowledge, MIT Press, Cambridge, MA.

[27] Stahl, G. (2008) Book review: Exploring thinking as communicating in CSCL, International Journal of Computer-Supported Collaborative Learning (ijCSCL), 3 (3), pp. 361-368.

[28] Stahl, G. (Ed.) (2009) Studying virtual math teams, Springer, New York, NY.

[29] Teasley, S. D. \& Roschelle, J. (1993) Constructing a joint problem space: The computer as a tool for sharing knowledge. In S. P. Lajoie \& S. J. Derry (Eds.), Computers as cognitive tools, Lawrence Erlbaum Associates, Inc., Mahwah, NJ, pp. 229-258.

[30] Vygotsky, L. (1930/1978) Mind in society, Harvard University Press, Cambridge, MA.

[31] Wegner, D. (1986) Transactive memory: A contemporary analysis of the group mind. In B. Mullen \& G. R. Goethals (Eds.), Theories of group behavior, Springer Verlag, New York, NY, pp. 185-208.

[32] Zhou, N., Zemel, A., \& Stahl, G. (2008) Questioning and responding in online small groups engaged in collaborative math problem solving, In: Proceedings of International Conference of the Learning Sciences (ICLS 2008), Utrecht, Netherlands. 